\title{
Comparative Study of Hematological Parameters in the Third Trimester of Hypertensive and Normotensive Pregnant Women
}

\author{
Md Eajaz Ahmed Shariff ${ }^{*}$, Fahd Al- Qahtani ${ }^{2}$, Modii Al-Qahtani ${ }^{3}$ \\ ${ }^{1}$ Division of Physiology Department of Basic Medical sciences Faculty of Dentistry Al-Baha University-65527 Kingdom of Saudi Arabia \\ ${ }^{2}$ Dean College of Dentistry Al-Baha University-65527 Kingdom of Saudi Arabia \\ ${ }^{3}$ MBBS student, Faculty of Medicine Al-Baha University Kingdom of Saudi Arabia
}

DOI: $\underline{10.36348 / \mathrm{sjpm} .2020 . \mathrm{v} 05 \mathrm{i} 09.004}$

| Received: 08.09.2020 | Accepted: 19.09.2020 | Published: 24.09.2020

*Corresponding author: Md Eajaz Ahmed Shariff

\section{Abstract}

Pregnancy induced hypertension (PIH) is a serious life threatening complication, resulting in intrauterine growth retardation (IUGR) of the fetus. The study was aimed to compare the hematological and during third trimester in hypertensive and normotensive pregnant women. It is a cross-sectional study undertaken at Dr BR Ambedkar Medical College and K.C.General Hospital Bangalore. Total of 43 pregnant women of which 19 were hypertensive and 24 were normotensive mothers were considered for the study. Women who had hypertension in pregnancy after the 20 weeks of gestation, with SBP and DBP of 140 and $90 \mathrm{~mm} \mathrm{Hg}$, with or without edema and or proteinuria were included. Hematological parameter were estimated by using Sysmex XN-9100 ${ }^{\mathrm{TM}}$ Automated Hematology System . The mean hemoglobin level of the hypertensive group $(8.13 \pm 0.5)$ was significantly lower than that of the normotensive group $(9.68 \pm 0.64)(\mathrm{p}<0.05)$. The mean platelet count of the hypertensive group $(164.15 \pm 34.8)$ was significantly lower than that of the normotensive group $(370.04 \pm 46.3)(\mathrm{p}<0.05)$. The mean lymphocytes level of hypertensive group $(1.29 \pm 5.6)$ was significantly lower than that of the normotensive group $(1.93 \pm 1.4)(\mathrm{p}<0.05)$. The mean WBC level of hypertensive group $(15.9 \pm 2.09)$ was significantly high than normotensive group $(7.8 \pm 1.7)(\mathrm{p}<0.05)$. The mean hematocrit level of hypertensive group $(31.10 \pm 2.55)$ was significantly high than normotensive group $(27.6 \pm 2.05)(p<0.05)$.Platelet count could be a suitable indicator for monitoring patients with PIH at risk of IUGR and Intra uterine death (IUD) of the fetus. Anemia may be associated with IUGR and IUD in PIH patients.

Keywords: Pregnancy induced hypertension (PIH), hematological parameters, and normotensive pregnant women.

Copyright @ 2020: This is an open-access article distributed under the terms of the Creative Commons Attribution license which permits unrestricted use, distribution, and reproduction in any medium for non-commercial use (NonCommercial, or CC-BY-NC) provided the original author and sources are credited.

\section{INTRODUCTION}

Hypertensive disorders of pregnancy remain major cause of maternal mortality and morbidity worldwide, it accounts to $8.0 \%$ of the maternal deaths. In women with pre-eclampsia, a history of symptoms obtained antenatally, with the key alarming clinical symptoms being headache, vomiting, visual disturbance and epigastric pain [1]. The association between proteinuria and pre-eclampsia has long been established and its presence is diagnostic of the disease and is indicative of its multisystem nature and leading to maternal and fetal morbidity and mortality. However, recent studies on pre-eclampsia with varying levels of proteinuria have pointed out that estimation of levels of proteinuria in women with pre-eclampsia do not $[2,3]$ correlate with maternal and fetal outcomes. Thus urinary protein estimation may not be a clinically useful predictive test, disputing the practice of deciding on delivery based on the severity of proteinuria. Over the years, a lot of interest has been directed at studies on the role of serum uric acid, urea and creatinine in the [4, 5] pathogenesis of pregnancy induced hypertension. At the same time hypertensive disorders account for $21 \%$ [6] of cases of thrombocytopenia in pregnancy and the risk of anemia may also increase with the severity of [7] hypertensive disorders. Thus, though the roles of serum urea, uric acid and creatinine have been studied by [8] many researchers, there is a constant ongoing search for better predictors and prognostic factors to assess the progress and severity of the disease, hematological [9] parameters being one among them. Currently there are no individual screening tests that are reliable, valid, and economical [10]. With this background of knowledge, the present study was undertaken to evaluate the relevance of routinely done renal functions and hematological parameters in assessment of pregnancy induced hypertension. 


\section{MATERIAL AND METHODS Study Design}

This is a hospital based prospective, crossectional study conducted at Dr BR Ambedkar Medical College and K.C.General Hospital Bangalore. Forty three pregnant women attending regular antenatal checkups of which 19 were hypertensive (Hypertensive group) and 24 were healthy mothers (Normotensive group) gave birth to singleton babies. Written Informed consent was obtained from all the participants after explaining the study in detail and ensuring the confidentiality. The study protocol was approved by institutional human ethical committee. Complete medical, social and obstetric history was recorded to identify the confounders.

The mothers were examined clinically along with recording of relevant investigation reports. The women included in this study were recruited from outpatient department and from admission wards after 20 weeks of pregnancies. On development of preeclampsia and fulfilment of selection criteria they were enrolled in the Hypertensive group. The women representing the normotensive group were chosen from the women who fulfilled the inclusion criteria but did not develop pre-eclampsia. They were labelled as normotensive, with no proteinuria.

The women were informed about the procedure and a verbal informed consent was taken before taking the sample. While evaluating the results of the study, relevant clinical data was collected from every patient, who included detailed history, general, systemic and obstetric examinations; baseline investigations including ultrasonography were performed. Blood pressure was measured in the right arm in semi recumbent position with the arm at the level of heart using a mercury sphygmomanometer.

\section{Hematological investigation}

The blood sample was taken from the antecubital vein. Two milliliter of blood sample was drawn aseptically using the $5 \mathrm{ml}$ syringe from the median ante cubital vein of all the case and control participants into EDTA-anticoagulated tubes. Hemoglobin, hematocrit, platelets, WBC, and lymphocytes were counted using Sysmex XN-9100 TM Automated Hematology System. Random blood sugar, blood urea, serum creatinine, uric acid and urinary protein estimation were done by standard laboratory techniques.

\section{Inclusion criteria}

- Patients having pre-eclampsia. As defined and classified by standard criteria of the

- American College of Obstetricians and Gynecologists

- Non-smoker Primigravida with Singleton pregnancy

- $\quad$ Age between 20 and 36 years
- Gestation age: After 20weeks onwards (calculated from the first day of last menstrual period).

\section{Exclusion criteria}

- Multiple pregnancies.

- Previous history of hypertension and proteinuria before conception or before 20 weeks of gestation.

- History of systemic illnesses like diabetes mellitus, renal disease, liver diseases.

- Eclampsia.

- History of hypertension without proteinuria

- Women with history of recent blood transfusion.

\section{STATISTICAL ANALYSIS}

Data obtained was tabulated and analysed by SPSS programme and Graph pad prism version 5.0 analysis of variance (ANOVA) and student' $t$ ' test were used for analysis. The level of significance of $(p<0.05)$ were considered significant.

\section{RESULTS}

Total number of the mothers participated in the study were 43 of which 19 mothers were hypertensive $(44.1 \%)$ and 24 mothers were normotensive $(55.9 \%)$.The mean age of hypertensive and normotensive mothers were $29.9 \pm 3.50$ and $28.01 \pm$ 4.48 respectively. There was no statistical difference between these groups $(\mathrm{p}>0.05)$. The mean maternal BMI $\left(\mathrm{kgs} / \mathrm{m}^{2}\right)$ in hypertensive and normotensive mothers were $26.63 \pm 2.64$ and $25.09 \pm 2.50$. The mean SBP and DBP in hypertensive and normotensive mother's were $158 \pm 7.90,98 \pm 5.16$ and $117.58 \pm 6.93$ and $5.5 \pm 1.04$ respectively. Urine protein in hypertensive and normotensive others were found to be $7.08 \pm 1.56$ and $5.5 \pm 1.04$. Blood pressure and Urine protein were significantly higher in the hypertensive mothers compared to normotensive others. There was statistical significance was noted between these groups ( $\mathrm{p}<0.05)$ [Table 1]. Among the renal parameters measured serum creatinine did not differ significantly ( $p>0.05$ ) between the groups which blood urea was significantly higher in hypertensive group versus normotensive group $(\mathrm{p}<0.05)$. Serum uric acid level was higher in hypertensive group in comparison to normotensive group $(\mathrm{p}<0.05)$.

The mean platelet count (thousands/ml) in hypertensive and normotensive mothers were found to be $164.15 \pm 34.8$ and $370.04 \pm 46.3$ respectively [Table2, Fig 1]. Mean platelet count was found to be lower in hypertensive group vs normotensive group. There was a statistically significance between hypertensive and normotensive mothers $(\mathrm{p}<0.05)$.

Hemoglobin concentration (gm \%) in hypertensive and normotensive mothers were $8.13 \pm 0.5$ 
and $9.68 \pm 0.64$ respectively. There statistically lower values in hypertensive mothers in comparison to the normotensive mothers [Table 2, Figure 2].Hematocrit $(\%)$ volume was found to be $31.10 \pm 2.55$ and, $27.6 \pm 2.05$ in hypertensive and normotensive mothers respectively.

There statistically higher values in hypertensive mothers in comparison to the normotensive mothers.[Table 2, Fig 2].There was a statistically significance between hypertensive and normotensive mothers $(\mathrm{p}<0.05)$.

Total WBC count (thousands/ml) and Lymphocyte count (thousands/ml) in hypertensive and normotensive mothers were found to be $15.9 \pm 2.09$, $1.29 \pm 5.6$ and $7.8 \pm 1.7,1.93 \pm 1.4$ respectively [Table 2, Fig 3]. Total WBC count (thousands/ml) and Lymphocyte count (thousands/ml) in hypertensive and normotensive mothers were found to be $15.9 \pm 2.09$, $1.29 \pm 5.6$ and $7.8 \pm 1.7,1.93 \pm 1.4$ respectively. Total WBC count was higher in the hypertensive mothers in comparison to normotensive mothers and it was statistically significant $(\mathrm{p}<0.05)$.Lymphocyte count was significantly lower in the hypertensive mothers in compared to normotensive mothers. There was no statistical correlation between these group $(\mathrm{p}>0.05)$.

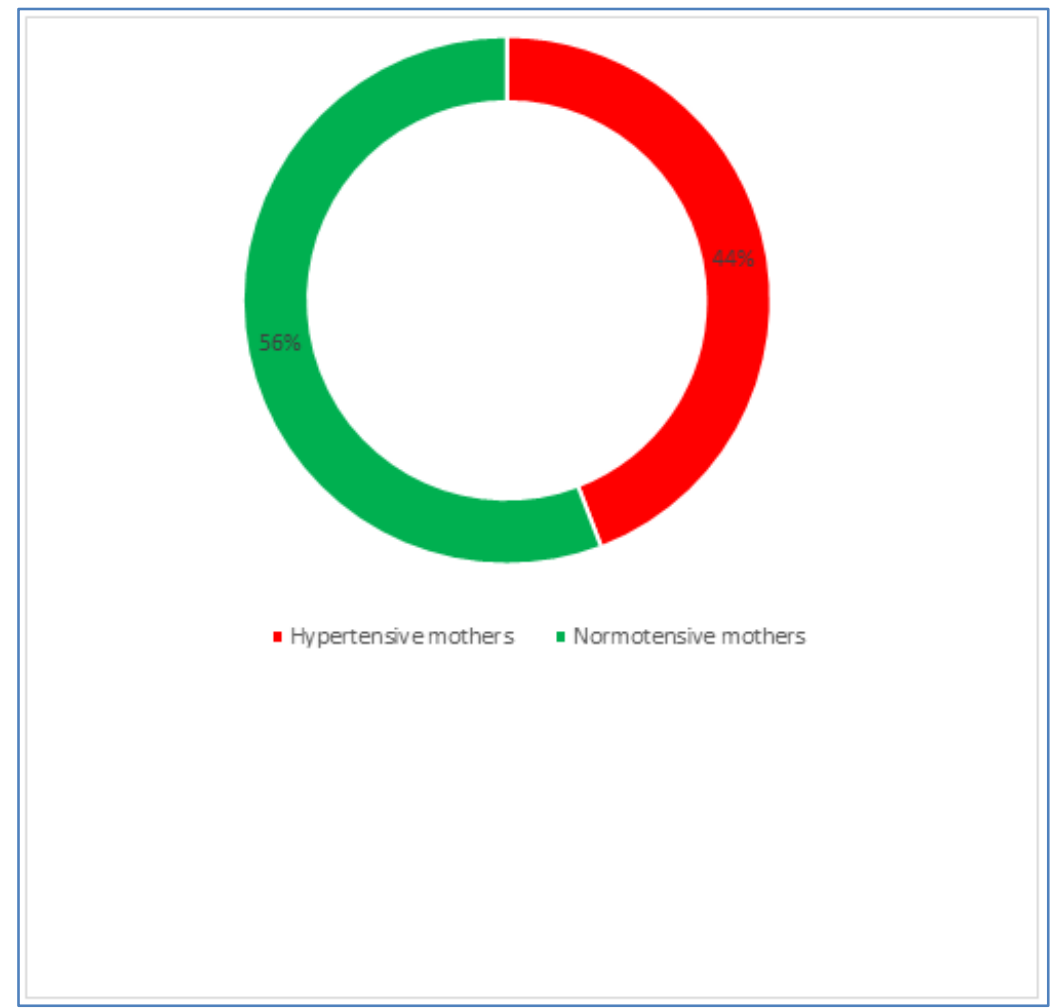

Fig-1: Percentage distribution of hypertensive and normotensive mothers

Table-1: Baseline and Renal parameters in Hypertensive Vs Normotensive mothers

\begin{tabular}{|l|c|c|c|}
\hline Parameters & Hypertensive mothers & $\begin{array}{c}\text { Normotensive } \\
\text { mothers }\end{array}$ & Level of significance \\
\hline & $(\mathrm{n}=19)$ & $(\mathrm{n}=24)$ & \\
\hline Maternal Age & $29.9 \pm 3.50$ & $28.01 \pm 4.48$ & $\mathrm{p}>0.05$ \\
\hline BMI(kgs/m2) & $26.63 \pm 2.64$ & $25.09 \pm 2.50$ & $\mathrm{p}>0.05$ \\
\hline $\mathrm{SBP}(\mathrm{mmHg})$ & $158 \pm 7.90$ & $117.58 \pm 6.93$ & $\mathrm{p}<0.05$ \\
\hline DBP(mmHg) & $98 \pm 5.16$ & $72.74 \pm 4.84$ & $\mathrm{p}<0.05$ \\
\hline Random Blood Sugar (mg \%) & $113.9 \pm 30.37$ & $102.61 \pm 21.43$ & $\mathrm{p}>0.05$ \\
\hline Urine Protein & $7.08 \pm 1.56$ & $5.5 \pm 1.04$ & $\mathrm{p}<0.05$ \\
\hline Creatinine (mg \%) & $0.67 \pm 0.18$ & $0.61 \pm 0.15$ & $\mathrm{p}>0.05$ \\
\hline Urea (mg \%) & $27.5 \pm 16.26$ & $16.75 \pm 11.56$ & $\mathrm{p}<0.05$ \\
\hline Serum uric acid level & $5.63 \pm 1.61$ & $4.34 \pm 1.61$ & $\mathrm{p}<0.05$ \\
\hline
\end{tabular}


Table-2: Hematological Parameters in Hypertensive Vs Normotensive mothers

\begin{tabular}{|l|l|l|l|}
\hline \multicolumn{1}{|c|}{ Hematological Parameters } & Hypertensive mothers & $\begin{array}{c}\text { Normotensive } \\
\text { mothers } \\
(\mathrm{n}=24)\end{array}$ & $\begin{array}{c}\text { Level of } \\
\text { Significance }\end{array}$ \\
\hline Hb Concentration $(\mathrm{gm} \%)$ & $(\mathrm{n}=19)$ & $9.68 \pm 0.64$ & $\mathrm{p}<0.05$ \\
\hline Hematocrit $(\%)$ & $2.13 \pm 0.5$ & $27.6 \pm 2.05$ & $\mathrm{p}<0.05$ \\
\hline Total WBC Count(thousands/ml) & $15.9 \pm 2.09$ & $7.8 \pm 1.7$ & $\mathrm{p}<0.05$ \\
\hline Lymphocyte count(thousands/ml) & $1.29 \pm 5.6$ & $1.93 \pm 1.4$ & $\mathrm{p}>0.05$ \\
\hline Platelet count(thousands/ml) & $164.15 \pm 34.8$ & $370.04 \pm 46.3$ & $\mathrm{p}<0.05$ \\
\hline
\end{tabular}

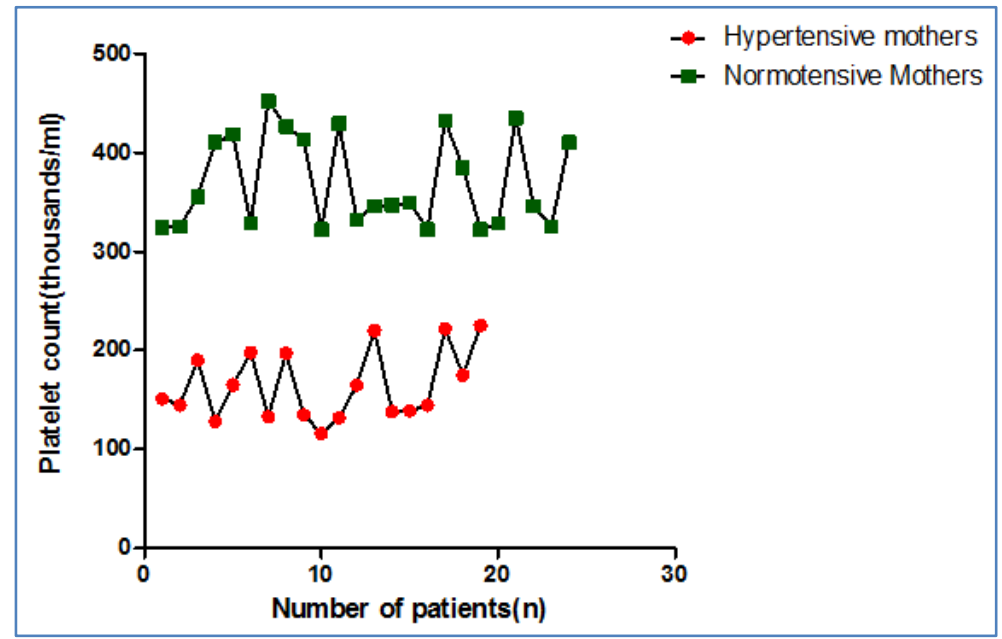

Fig-1: Platelet count in hypertensive vs normotensive mothers

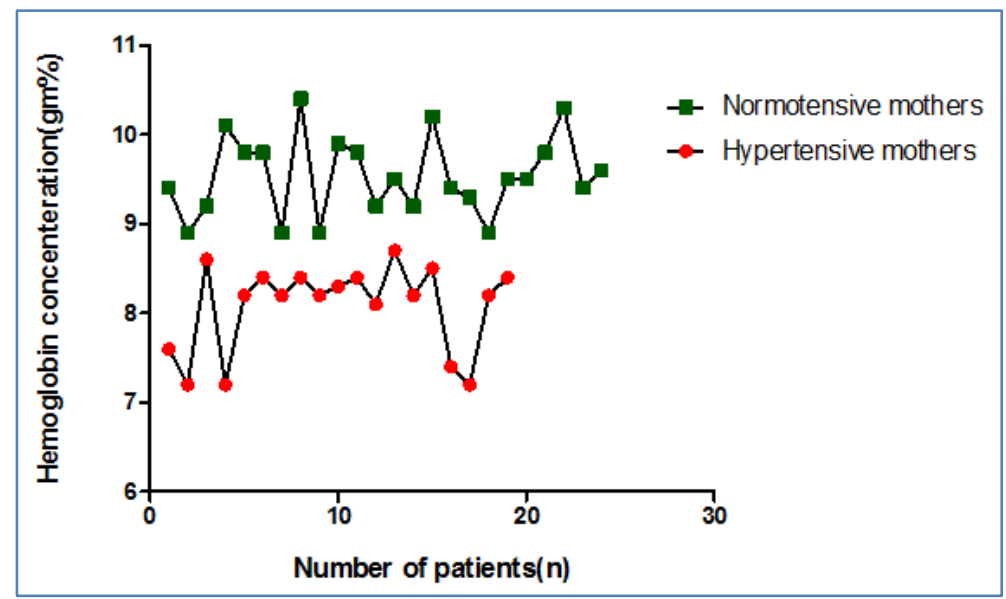

Fig-2: Hemoglobin concenteration in hypertensive vs normotensive mothers

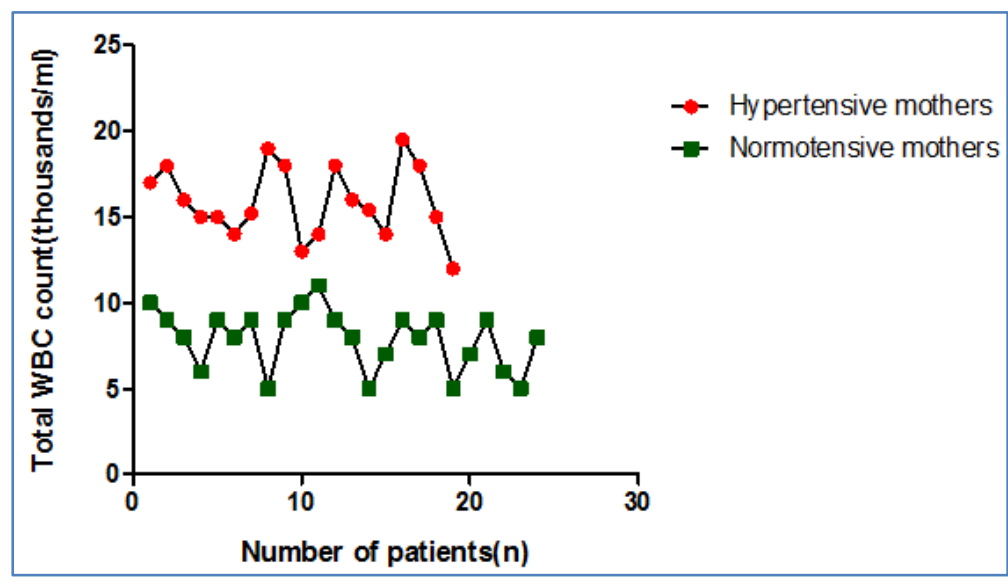

Fig-3: Total WBC count in hypertensive vs normotensive mothers 


\section{DISCUSSION}

Pregnancy induced hypertension poses greater risk of IUGR and IUD of foetus. But currently there are no appropriate indicators in monitoring the advancement of PIH leading to IUGR and IUD of foetus. We evaluated the significance of renal and hematological parameters in PIH mothers. In the present study, systolic and diastolic blood pressure was significantly higher in hypertensive subjects compared to normotensive subjects. But blood pressure of hypertensive subjects with normal foetus did not vary compared to PIH mothers with IUGR and IUD of foetus. Our finding was in concordance with the findings of Cnossen et al. who also have observed that systolic and diastolic blood pressures are poor predictors of [11] pre-eclampsia. However in their study pre-eclampsia was reflected, unlike as PIH mothers with IUGR and IUD of foetus. In the present study, urine protein was significantly higher in hypertensive compared to normotensive subjects. But hypertensive subjects with and without IUGR and IUD of foetus were comparable with regard to urine protein. This finding suggests that although raised protein is a characteristic feature of PIH, this parameter may not be involved in the advancement of pathogenesis of PIH leading to IUGR and IUD of foetus. There was no correlation between estimation of levels of proteinuria with maternal and foetal outcome in women with pre[1-3] eclampsia as reported by Lao TT et al. and Newman MG et al. Our finding was in concordance with the findings of Lao TT et al. and Newman MG et al. In the present study, serum uric acid was significantly higher in hypertensive subjects compared to normotensive subjects [Table 2]. Findings of previous research work on association between elevated serum uric acid levels and PIH is conflicting. Wake VC et al. have observed that in subjects with pre-eclampsia plasma uric acid level will help to predict those that will develop [12] eclampsia. Elevated Serum uric acid levels in hypertensive pregnancy remains an important finding because it identifies women at increased risk of adverse foetal outcome even women with gestational hypertension without any other features of [13] preeclampsia as documented by Hawkins A et al. High positive correlation was observed between levels of serum uric acid and severity of PIH in relation to hypertension and [14] proteinuria as reported by Mustaphi R et al. However, Hickman PE et al. have noted serum uric acid as an unreliable indicator of developing [15] hypertension in the individual women. Salako BL et al. have reported that single estimation of serum uric acid level early in pregnancy are of little value in the prediction [15] of pre-eclampsia. However, our study finding suggests that with PIH, uric acid metabolism may get impaired but it may not be linked with pathogenesis of the disorder review and metaanalysis.
Our findings revealed mean platelet count was found to be lower in hypertensive group vs normotensive group. There was a statistically significance between hypertensive and normotensive mothers $(\mathrm{p}<0.05)$. Our finding is indicating that low platelet count is more apparent during 3rd trimester of pregnancy. The variation in platelet count among pregnant women with PIH may be due to an increased consumption with reduced life span and increased aggregation by increased levels of thromboxane A2 at placental circulation.10 Thromboxane A2 promotes vasospasm, induces supplementary platelet aggregation and endothelial damage, which add an important contribution to maintaining platelet dysfunction and promoting platelet consumption (activation, aggregation, microangiopathic hemolysis induced by severe vasospasm), resulting in thrombocytopenia. It might also be due to incomplete trophoblastic inversion of the uterine spiral arteries resulting to placental ischemia followed by release of anti-angiogenic proteins that lead to endothelial dysfunction [16].

In our study hemoglobin concentration (gm\%) statistically lower values in hypertensive mothers in comparison to the normotensive mothers. A retrospective study done in singleton mothers including 75 subjects with PIH. Zhang et al. have reported that the risk of anemia increases with the severity of hypertensive disorders [21].

Our findings were hematocrit $(\%)$ volume was statistically lower values in hypertensive mothers in comparison to the normotensive mothers. A study which shows PCV, hemoglobin and platelets concentration was significantly lower in preeclampsia as compared to normal pregnancy [19]. In our study the total WBC count (thousands/ml) and Lymphocyte count (thousands $/ \mathrm{ml}$ ) in hypertensive and normotensive mothers Total WBC count was higher in the hypertensive mothers in comparison to normotensive mothers and it was statistically significant $(\mathrm{p}<0.05)$.Lymphocyte count was significantly lower in the hypertensive mothers in compared to normotensive mothers. There was no statistical correlation between these group ( $\mathrm{p}>0.05)$. Lurie et al. [18] Conducted a study which shows that absolute neutrophil count was significantly elevated in pre-eclamptic patients and absolute lymphocyte counts declined in patients with preeclampsia as compared with uncomplicated pregnancy and increase in neutrophils count was reported in severe preeclampsia indicating an inflammatory response to the fetus or infection in the environment. The significant difference found in the $\mathrm{T}$ lymphocyte subsets in the peripheral blood of pregnant women with and without preeclampsia could be as a result of the immunosuppressive effect of placental products such as pregnancy specific beta-1 glycoprotein, progesterone or human chorionic gonadotrophin [19]. 


\section{CONCLUSION}

The hematological parameters measured, decreased platelet count may be an appropriate marker in monitoring subjects with PIH at risk of IUGR and IUD of foetus. Reduced hemoglobin and packed cell volume may be associated with IUGR and IUD of foetus in PIH mothers. Proteinuria, uricemia, higher ESR, higher total leucocyte count, higher neutrophil, monocyte and basophil counts are associated with PIH but does not reflect on PIH mothers at risk of IUGR and IUD of foetus.

\section{ACKNOWLEDGEMENT}

I extend my thanks and gratitude to the Doctors, Nurses of K.C General Hospital for all the technical support throughout the study

\section{REFERENCES}

1. Lao, T. T., Chin, R. K. H., \& Lam, Y. M. (1988). The significance of proteinuria in pre-eclampsia; proteinuria associated with low birth weight only in pre-eclampsia. European Journal of Obstetrics \& Gynecology and Reproductive Biology, 29(2), 121127.

2. Newman, M. G., Robichaux, A. G., Stedman, C. M., Jaekle, R. K., Fontenot, M. T., Dotson, T., \& Lewis, D. F. (2003). Perinatal outcomes in preeclampsia that is complicated by massive proteinuria. American journal of obstetrics and gynecology, 188(1), 264-268.

3. Thangaratinam, S., Coomarasamy, A., O'Mahony, F., Sharp, S., Zamora, J., Khan, K. S., \& Ismail, K. M. (2009). Estimation of proteinuria as a predictor of complications of pre-eclampsia: a systematic review. BMC medicine, 7(1), 1-9.

4. Egwuatu, V. E. (1986). Plasma concentration of urate, urea and creatinine in Nigerian primigravidae with pre-eclampsia. Tropical and geographical medicine, 38(1), 11-15.

5. Mustaphi, R., Gopalan, S., Dhaliwal, L., \& Sarkar, A. K. (1996). Hyperuricemia and pregnancy induced hypertension--reappraisal. Indian journal of medical sciences, 50(3), 68-71.

6. Shehata, N., Burrows, R., \& Kelton, J. G. (1999). Gestational thrombocytopenia. Clinical obstetrics and gynecology, 42(2), 327-334.

7. Zhang, Q., Li, Z., \& Ananth, C. V. (2009). Prevalence and risk factors for anaemia in pregnant women: a population- based prospective cohort study in China. Paediatric and Perinatal Epidemiology, 23(4), 282-291.

8. Manjareeka, M., \& Nanda, S. (2013). Elevated levels of serum uric acid, creatinine or urea in preeclamptic women. Int $J$ Med Sci Public Health, 2(1), 43-47.

9. Sivakumar, S., Bhat, B. V., \& Badhe, B. A. (2007). Effect of pregnancy induced hypertension on mothers and their babies. The Indian Journal of Pediatrics, 74(7), 623-625.

10. Levine, R. J., \& Lindheimer, M. D. (2009). Firsttrimester prediction of early preeclampsia: a possibility at last!.

11. Cnossen, J. S., Vollebregt, K. C., De Vrieze, N., Ter Riet, G., Mol, B. W., Franx, A., ... \& Van Der Post, J. A. (2008). Accuracy of mean arterial pressure and blood pressure measurements in predicting pre-eclampsia: systematic review and meta-analysis. Bmj, 336(7653), 1117-1120.

12. Wakwe, V. C., \& Abudu, O. (1999). Estimation of plasma uric acid in pregnancy induced hypertension (PIH). Afr J Sci, 155-158.

13. Hawkins, T. A., Roberts, J. M., Mangos, G. J., Davis, G. K., Roberts, L. M., \& Brown, M. A. (2012). Plasma uric acid remains a marker of poor outcome in hypertensive pregnancy: a retrospective cohort study. BJOG: An International Journal of Obstetrics \& Gynaecology, 119(4), 484-492.

14. Mustaphi, R., Gopalan, S., Dhaliwal, L., \& Sarkar, A. K. (1996). Hyperuricemia and pregnancy induced hypertension--reappraisal. Indian journal of medical sciences, 50(3), 68-71.

15. Hickman, P. E., Michael, C. A., \& Potter, J. M. (1982). Serum Uric Acid as a Marker of Pregnancy- Induced Hypertension. Australian and New Zealand Journal of Obstetrics and Gynaecology, 22(4), 198-202.

16. Salako, B. L., Odukogbe, A. T. A., Olayemi, O., Adedapo, K. S., Aimakhu, C. O., Alu, F. E., \& Ola, B. (2003). Serum albumin, creatinine, uric acid and hypertensive disorders of pregnancy. East African medical journal, 80(8), 424-428.

17. Karim, R., Sacher, R. A. (2004). Thrombocytopenia in pregnancy. curr. Hematology Rep, 3(2):128-33.

18. Lurie, S., Frenkel, E., \& Tuvbin, Y. (1998). Comparison of the differential distribution of leukocytes in preeclampsia versus uncomplicated pregnancy. Gynecologic and obstetric investigation, 45(4), 229-231.

19. Onyemelukwe, G. C., Ekwempu, C. C., \& Alexander, L. C. (1985). Pregnancy- specific $\beta$ glycoprotein (SP1) in normal pregnancy in Nigeria. International Journal of Gynecology \& Obstetrics, 23(4), 347-349.

20. Monteiro, G., Subbalakshmi, N. K., \& Pai, S. R. (2014). Relevance of measurement of hematological parameters in subjects with pregnancy induced hypertension. Nitte University Journal of Health Science, 4(1), 15.

21. Zhang, Q., Li, Z., \& Ananth, C. V. (2009). Prevalence and risk factors for anaemia in pregnant women: a population- based prospective cohort study in China. Paediatric and Perinatal Epidemiology, 23(4), 282-291. 